\title{
Effect of Vitamin D Supplement Consumption on Muscle Strength, Muscle Function and Body Composition in Vitamin D-deficient Middle-aged Women: A Randomized Clinical Trial
}

\author{
Behnaz Abiri ${ }^{1}$, Mohammad Reza Vafa ${ }^{2}$, Mohsen Dehghani ${ }^{3}$, Nazanin Moslehi ${ }^{4}$ Javad Sarrafzadeh $^{5}$ \\ 1- M.Sc of Nutrition Sciences, Department of Nutrition, School of Public Health, Iran University of Medical Sciences, Tehran, Iran \\ 2- Department of Nutrition, School of Public Health, Iran University of Medical Sciences, Tehran, Iran \\ 3- Ph.D Student in Epidemiology, Department of Epidemiology, School of Public Health, Iran University of Medical Sciences, Tehran, Iran \\ 4- Ph.D Student in Nutrition, Nutrition and Endocrine Research Center, Research Institute for Endocrine Sciences, Shahid Beheshti University of \\ Medical Sciences, Tehran, Iran \\ 5- Department of Physical Therapy, School of Rehabilitation Sciences, Iran University of Medical Sciences, Tehran, Iran
}

\section{A B S T R A C T}

Background and Objectives: Sarcopenia is explained as the loss of muscle strength and muscle mass with aging, and is one of the major risk factors for metabolic diseases. Cross-sectional studies demonstrate that vitamin $\mathrm{D}$ is associated with sarcopenia in both men and women. The aim of this study was to investigate the effect of vitamin D supplement consumption on muscle strength, muscle function and body composition in middle-aged women.

Materials and Methods: In this randomized double-blind placebo-controlled trial, 71 women aged 40-55 years old, with the serum $25(\mathrm{OH})-\mathrm{D}<25 \mathrm{ng} / \mathrm{ml}$, were divided in two groups randomly, and received a $1000 \mathrm{IU}$ vitamin $\mathrm{D}$ tablet $(\mathrm{n}=37)$ or a placebo $(\mathrm{n}=34)$, daily for 12 weeks. At the beginning and at the end of the study, dietary intake, anthropometric indices, muscle strength and function, serum 25(OH)-D, physical activity level and sun exposure were assessed. Statistical analysis was performed with SPSS version 20.

Results: A significant difference in serum 25(OH)-D was found between the intervention and placebo groups at the end of the study $(\mathrm{P}<0.001)$. In the vitamin $\mathrm{D}$ group, handgrip strength was improved, while hand grip strength $(\mathrm{P}=0.233)$ and knee extension strength $(\mathrm{P}=0.337)$ between the two groups did not differ significantly after 12 weeks. The mean of timed get up and go test, decreased significantly in the vitamin $\mathrm{D}$ group compared to the controls $(\mathrm{P}<0.001)$. Within the intervention group, body fat content decreased significantly after 12 weeks $(\mathrm{P}<0.001)$, but did not result a significant difference between the two groups $(\mathrm{P}=0.051)$.

Conclusions: It seems in this vitamin D-deficient middle-aged women group, 1000 IU vitamin D consumption daily for 12 weeks resulted in improvement of muscle function and body composition, but had no significant effect on muscle strength.

Keywords: Vitamin D, Muscle strength, Muscle function, Middle-aged women

\section{Introduction}

Aging is related to the slow loss of muscle strength and muscle mass, resulting in functional impairements, disability, and metabolic disorders $(1,2)$, and exert a notable burden on individuals and community. This age-related loss of muscle strength is more important public health concern for women because of earlier development of muscular weakness, long life, and exhibiting higher rates of disablements (3). Reducing in muscle strength in some degree is part of normal aging; however, it can be delayed or accelerated by some factors including nutritional status, overweight, and physical activity 
(2). Different rates of decreasing in muscle strength in the population also established the important effects of modifiable behavioral factors on muscle strength (4). Existing studies, which are mainly observational, show the potential effects of dietary patterns (5), quality of diet, and especially intakes of protein, vitamin $\mathrm{D}$, antioxidants, and nutrients on muscle strength (4).

Vitamin D is a fat soluble vitamin that has an essential role in calcium homeostasis and keeping of normal bone metabolism (6). 25-hydroxyvitamin D is the major form in the circulation and is the primary index of vitamin D status in humans (6). Data propose that vitamin D status is important for the different organs such as pancreatic $\beta$-cells, vascular endothelial cells, neurons, immune cells, osteoblasts and myocytes; all of these contain vitamin D receptors (6). Moreover, vitamin D deficiency is associated with loss of skeletal muscle mass and function (6). In both a cross-sectional study and a prospective study, low serum levels of 25-hydroxyvitamin D was associated with an increased risk of sarcopenia, regardless of obesity $(7,8)$.

Vitamin D deficiency is related to muscular weakness, and is usual in elderly people $(8,9)$. Older people are susceptible to develop vitamin D deficiency because of reduced dietary intake, diminished sun exposure, decreased skin thickness, reduced intestinal absorption, and impaired hydroxylation in the liver and kidneys (11-13).

Vitamin D metabolites influence on muscle metabolism in three ways: 1)by mediating gene transcription, 2)quick pathways that not involving DNA synthesis, and 3)by the allelic variant of the vitamin receptor (15).

Vitamin D supplementation induces fast changes in calcium metabolism of the muscle cells that cannot be explained by slow genetic pathways. Evidence demonstrates that $1,25(\mathrm{OH}) \mathrm{D}_{3}$, possibly through the vitamin D membrane receptor $(15,16)$, influences directly on the muscle cell membrane. On $1,25(\mathrm{OH}) \mathrm{D}_{3}$ binding, several second-messenger pathways were activated in the muscle cell, leading to raised calcium uptake (within minutes) that releases the activatad calcium channels through both voltagedependent calcium channels $(17,18)$ and calcium $(19)$.
Also low serum 1,25(OH)-D level may lead to overweight because vitamin $\mathrm{D}$ is a major determinant of PTH level. Elevated PTH level promotes calcium influx into adipocytes (20), where intracellular calcium enhances lipogenesis; therefore, high PTH level may be associated with weight gain (20).

Hence, the present study aimed to investigate the effect of vitamin D supplementation on body composition, muscle strength, and functional mobility in apparently healthy middle-aged women.

\section{Materials and Methods}

This study was a randomized, double-blinded and placebo-controlled trial. Participants were recruited from staff at Iran University of Medical Sciences (IUMS). Volunteers could participate in the study if they were female, aged 40-55 years with the serum $25(\mathrm{OH})-\mathrm{D}<25 \mathrm{ng} / \mathrm{ml}$, had a body mass index (BMI) $18.5-29.9 \mathrm{~kg} / \mathrm{m}^{2}$, were not suffering from any chronic diseases, were not a smoker, non- pregnant or lactating or menopause, and were not taking vitamin D supplement, laxative, or hormone medications. We excluded the women who had less than $80 \%$ compliance with the treatment, and those who were participating in weight loss programmes or taking nutritional supplements.

The number of participants calculated for each group was 31 at $80 \%$ power and $\alpha$ of 0.05 to defect a difference of $4 \mathrm{~kg}$ in handgrip strength between the groups with an SD of $5.6 \mathrm{~kg}$ (21). To allow for attrition, 37 participants were recruited for each group.

The 74 participants, who met the criteria, were randomly allocated in to two groups: vitamin D group $(\mathrm{n}=37)$ had to take a vitamin D supplement tablet of 1000 IU (Jalinous; made in Iran), and the placebo group $(n=34)$ to take one placebo tablet daily with lunch. The placebo was made of maltodextrin ("Roshd" Pharmaceutical Incubation Center of Tehran University of Medical Sciences) and had an appearance to the vitamin D tablet. The intervention was conducted for 12 weeks. The participants were asked not to change their routine dietary intake and physical activity during the intervention.

The present study was approved by the Ethics Committee of the Iran University of Medical Sciences, and Iranian Registry of Clinical Trial 
(registration no. IRCT 201112182365N6), and written informed consent and subject assent were obtained.

The participants were allocated randomly using a random number table; for this, a person who was not involved in the study protocol created the randomization list and allotted the participants to the vitamin D or placebo group. Twenty eight vitamin D and placebo tablets were put in to unlabeled containers; the study leader labeled these containers with participant numbers using the randomization list. Three containers for each subject were provided for intervention during three 4-week periods. Compliance with the intervention was evaluated by pill counts. For this, all the participants returned their three containers at the end of the each 4-week period.

At the beginning and at the end of the study, serum 25(OH)-D, anthropometric, dietary intakes, sun exposure and physical activity variables were assessed.

Blood samples were gathered after $10 \mathrm{~h}$ overnight fasting before and after the intervention, and serum was obtained by centrifugation at 800-1000 RPM for $10 \mathrm{~min}$; the serum samples were frozen and stored at $80^{\circ} \mathrm{c}$. Serum $25(\mathrm{OH})-\mathrm{D}$ was measured by ELISA (Micro plate Reader MPR4 Plus, Hiperion) and Euro Immune Kit.

We assessed body weight to the nearest $0.1 \mathrm{~kg}$ while the participants were minimally clothed and without shoes by e-body (Beurer, Germany). Height was measured to the nearest $0.5 \mathrm{~cm}$ in standing position without shoes. Body mass index (BMI) was computed as body weight $(\mathrm{kg})$ divided by height squared $\left(\mathrm{m}^{2}\right)$. Body fat mass was taken by e-body (Beurer, Germany).

Dietary intakes were evaluated with a 24 -h food recall for 2 days (1 week day and 1 weekend day), and nutrient intakes were estimated using nutritionist 4 software. Physical activity level was assessed by the short form of the International Physical Activity Questionnaire (IPAQ) and expressed in MetMin/week (22). Sun exposure was assessed quantitatively with the questionnaire. A sunshine exposure score was acquired based on (a) how often and what time of day the subjects were outside, (b) which parts of the body were exposed as a percentage of total body surface area, and (c) did the subjects use sun screen or not (23).
Muscle Performance: We measured upper body strength and lower body strength by handgrip strength test and knee extension strength test, respectively, and muscle function was assessed quantitatively using Time Get Up and Go test (TGUG) before and after the intervention.

Handgrip strength was evaluated in $\mathrm{kg}$ in dominant hand using a hand-held dynamometer (digital hand dynamometer "DIGI-II," Korea). The participants were seated on standard armchair with shoulder adducted and neutrally rotated, elbow bent to $90^{\circ}$, and the forearm and wrist were in a neutral position (24).

Isometric knee extension strength was assessed with a calibrated hand held dynamometer (Nicholas Manual Muscle Tester; Lafayette Inc) -in $\mathrm{kg}$ in dominant leg. The participants were seated on straight-back standard chair with their hips and knees bent $90^{\circ}$. The dynamometer was put proximal to the ankle joint of subjects, and they were asked to raise their leg (25). During the testing, the subjects were encouraged to raise the force to the greatest slowly while the tester was opposing.

For TGUG test, the subjects were seated on the chair. They were asked to stand up and walk at normal step $3 \mathrm{~m}$, turn to chair, and sit down. Value in seconds (s) was used $(26,27)$.

Statistical analysis was performed with SPSS version 20. Descriptive statistics were presented as mean \pm SD . We examined the normality of data with Kolmogorov-Smirnov test. All data had been normally distributed. Paired t-test was used to compare baseline and after 12 weeks values in each group. Differences between the two groups at baseline, at week 12, and its changes after 12 weeks were examined with independent $\mathrm{t}$-test. $\mathrm{P}<0.05$ were considered significant statistically.

\section{Results}

Of 74 participants primarily included in the study, three in the placebo group withdrew because of noncompliance with the intervention, and personal reasons. Compliance with treatment was roughly $90 \%$ in each group. No significant differences between the groups were seen for age, weight, height, BMI, physical activity, and serum 25(OH)-D (Table 1). Moreover, no significant changes in weight, BMI, physical activity, and serum 25(OH)-D were observed within each group during the intervention. 
Behnaz Abiri, et al: Vitamin D supplement consumption and vitamin D-defficient middle-aged women

Table 1. Characteristics of the participants in the vitamin D and placebo groups at baseline and after 12 weeks

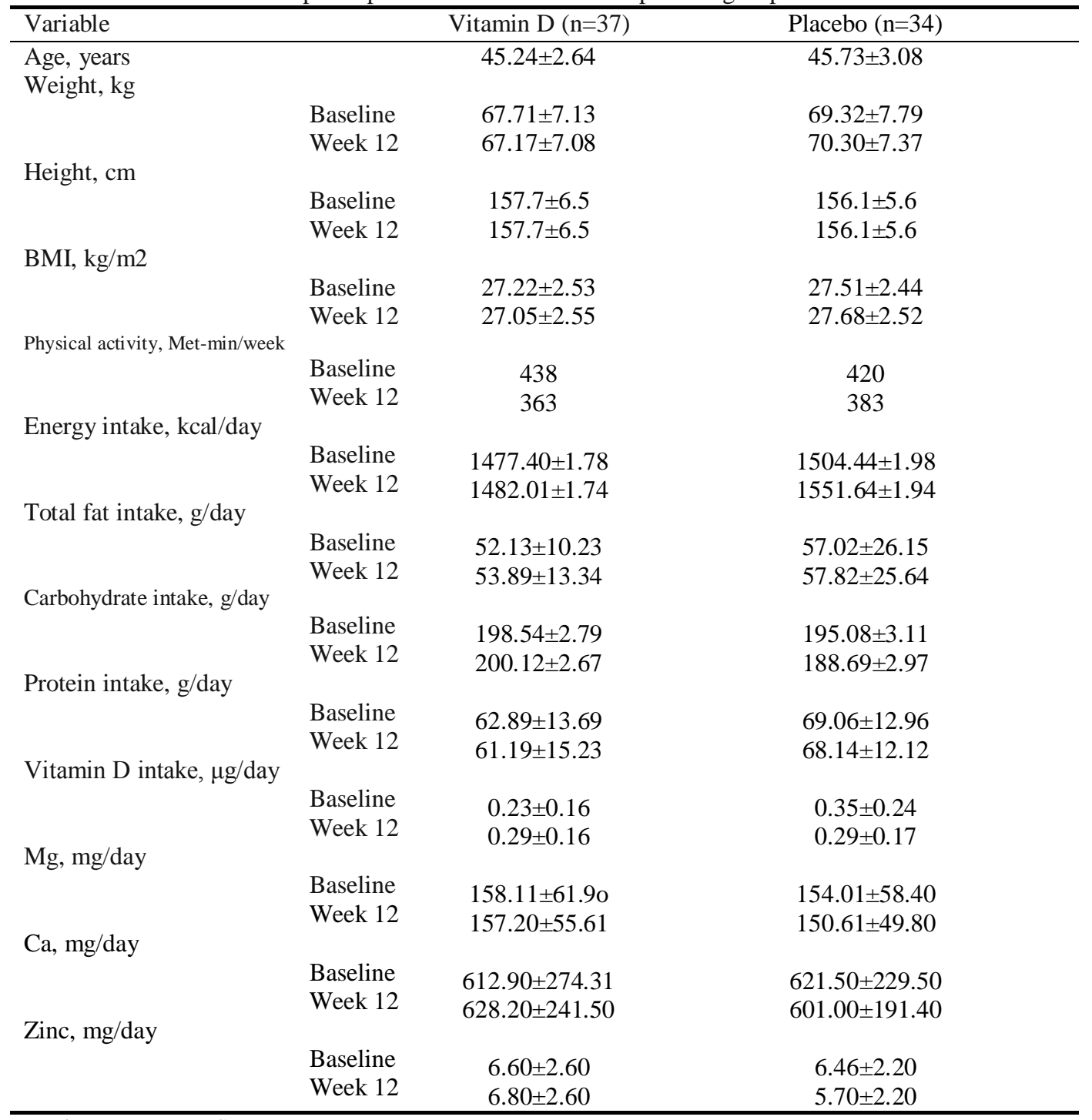

Values are mean \pm SDs

The mean dietary intake of energy, carbohydrate, protein, total fat, and vitamin $\mathrm{D}$, calcium, magnesium was not significantly different between the two groups at baseline and end of the study (Table 1). These dietary intake items did not alter significantly within each group during the intervention. In addition, this study was done in winter, and the participants mostly used sun screen with SPF >10; therefore in both the vitamin $\mathrm{D}$ and placebo groups at baseline and also during the intervention, cutaneous synthesis of vitamin $\mathrm{D}$ was not considerable.

Serum 25(OH)-D levels increased to $25.05 \pm 6.07$ vs. $13.33 \pm 6.22 \mathrm{ng} / \mathrm{ml} \quad(\mathrm{P}<0.001)$ respectively in vitamin $\mathrm{D}$ group, in comparison to the placebo group after 12 weeks (Table 2). Therefore, serum 25(OH)-D significantly increased in the intervention group compared to the placebo group.

There was no significant difference between the groups in relation to handgrip strength and knee extension strength at baseline and after 12-week intervention. Handgrip strength increased from $24.51 \pm 10.64$ to $24.60 \pm 4.84 \mathrm{~kg}(\mathrm{P}=0.960)$ in the vitamin D group and decreased from $24.05 \pm 5.86$ to $23.02 \pm 6.18 \mathrm{~kg}(\mathrm{P}=0.009)$ in the control group at the end of week 12 compared to baseline, but the increase did not significantly different between the two groups $(\mathrm{P}=0.233)$. Knee extension strength rose from $28.19 \pm 13.98$ to $26.03 \pm 4.80 \mathrm{~kg}$ in the vitamin D group, and from $24.77 \pm 6.45$ to $24.70 \pm 6.65 \mathrm{~kg}$ in the control group during the intervention, but none of these 
changes reached significant. The mean TGUG test reduced from $9.75 \pm 2.07$ to $8.27 \pm 1.74 \mathrm{~s}$ in the vitamin D group $(\mathrm{P}<0.001)$ as compared to baseline, while no significant change was observed in the placebo group (Table 2). A significant difference was observed in TGUG test between the two groups at week 12 $(\mathrm{P}<0.001)$.

There was no significant difference between the groups in fat mass at baseline. The body fat content significantly decreased from 26.00 \pm 4.96 to $25.20 \pm 4.79 \mathrm{~kg}(\mathrm{P}<0.001)$ in the intervention group, and increased from $26.94 \pm 4.57$ to $27.57 \pm 5.04 \mathrm{~kg}$ $(\mathrm{P}=0.183)$ in the placebo group at the end of week 12 . However, the changes did not reach significant compared with the placebo group $(\mathrm{P}=0.051)$. (Table 3).

Table 2. Comparison of the baseline and post treatment values (Mean \pm SD) in the vitamin $\mathrm{D}$ and placebo groups; serum $25(\mathrm{OH})-\mathrm{D}$, muscle strength and muscle function variables ${ }^{\mathrm{a}}$

\begin{tabular}{|c|c|c|c|c|c|c|}
\hline \multirow[t]{2}{*}{ Variable } & \multicolumn{2}{|c|}{ Baseline } & \multirow[t]{2}{*}{$\mathrm{P}$ value ${ }^{\mathrm{b}}$} & \multicolumn{2}{|c|}{ Week 12} & \multirow[t]{2}{*}{$\mathrm{P}$ value $^{\mathrm{b}}$} \\
\hline & Vitamin D & Placebo & & Vitamin D & Placebo & \\
\hline $25(\mathrm{OH})-\mathrm{D}_{3}(\mathrm{ng} / \mathrm{ml})$ & $15.72 \pm 6.68$ & $16.85 \pm 5.98$ & 0.710 & $25.05 \pm 6.07$ & $13.33 \pm 6.22$ & $<0.001^{*}$ \\
\hline Handgrip strength(kg) & $24.51 \pm 10.64$ & $24.05 \pm 5.86$ & 0.824 & $24.60 \pm 4.84$ & $23.02 \pm 6.18$ & 0.233 \\
\hline Knee extension strength $(\mathrm{kg})$ & $28.19 \pm 13.98$ & $24.77 \pm 6.45$ & 0.197 & $26.03 \pm 4.80$ & $24.70 \pm 6.65$ & 0.337 \\
\hline Time Get Up and Go test (s) & $9.75 \pm 2.07$ & $10.05 \pm 1.79$ & 0.515 & $8.27 \pm 1.74$ & $10.29 \pm 2.03$ & $<0.001^{*}$ \\
\hline
\end{tabular}

Table 3. Comparison of the baseline and post treatment values (Mean \pm SD) in the vitamin $\mathrm{D}$ and placebo groups; fat mass ${ }^{\mathrm{a}}$

\begin{tabular}{|c|c|c|c|c|c|c|}
\hline \multirow[t]{2}{*}{ Variable } & \multicolumn{2}{|c|}{ Vitamin D group } & \multirow[t]{2}{*}{$\mathrm{P}$ value $^{\mathrm{b}}$} & \multicolumn{2}{|c|}{ Placebo group } & \multirow[t]{2}{*}{$P$ value } \\
\hline & Baseline & Week 12 & & Baseline & Week 12 & \\
\hline $\begin{array}{c}\text { Fat } \\
\operatorname{mass}(\mathrm{kg})\end{array}$ & $26.00 \pm 4.96$ & $25.20 \pm 4.79$ & $<0.001^{*}$ & $26.94 \pm 4.57$ & $27.57 \pm 5.04$ & 0.183 \\
\hline
\end{tabular}

\section{Disc ussion}

In the present study, daily intake of 1000 IU vitamin $\mathrm{D}$ for 12 weeks significantly increased serum 25(OH)-D level in the vitamin D group as compared with the placebo group. But significantly improving vitamin D status in the intervention group did not result in significant difference in muscle strength between the two groups. In a study of 70 female geriatric patients $>65$ years of age with serum 25 hydroxyvitamin $\mathrm{D}_{3}$ levels between 20 and $25 \mathrm{nmol} / \mathrm{L}$, the clinical trial study to investigate cholecalciferol $400 \mathrm{IU} /$ day+calcium $500 \mathrm{mg} /$ day (D/Cal group) or a placebo+calcium $500 \mathrm{mg} /$ day (Plac/Cal group) for 6 months led to significant improvement in vitamin $\mathrm{D}$ status in the D/Cal group compared with the $\mathrm{Plac} / \mathrm{Cal}$ group; however, it did not result in a significant difference in muscle strength or muscle functional between the two groups (28). Evidence has found that vitamin D supplementation increased serum levels of 25(OH)-D to normal levels, more than $30 \mathrm{ng} / \mathrm{ml}$ or 75 $\mathrm{nmol} / \mathrm{L}$ (29), which was related to improvement in muscle strength and balance; this may express the basis of fall prevention with higher doses of vitamin D. Optimal fall prevention has been found in trials in which mean serum levels achieved in 75 to 100 $\mathrm{nmol} / \mathrm{L}$, whereas low doses of vitamin D ( $<800 \mathrm{IU}$ ) or achieved serum 25(OH)-D levels less than 60 $\mathrm{nmol} / \mathrm{L}$ did not reduce falls (30). Vitamin D deficiency is reported to cause proximal muscle weakness with a reduction in type 2 muscle fibers (31). Type 2 fibers are fast twitch and are involved in activities with high intensity but short duration. Moreover, several vitamin D receptor polymorphism, which are defined as subtle variations in DNA sequence of vitamin D receptor gene, exist that are 
associated with a range of biological features including muscle strength (31). Another barrier that leads to variability when attempting to describe the relationship between vitamin $\mathrm{D}$ and muscle strength in adults includes testing methods. Handgrip strength was one of the most common assessors for muscle strength but different equipments were utilized on different muscle groups to complete these measurements and possibly contribute to outcome variability (32).

The TGUG test is the time required to complete a series of important functional performances including stand up from a standard chair, walk $3 \mathrm{~m}$, turn down, and sit down, and is a beneficial method for assessment of functional mobility $(26,27)$. It caused a significant decrease in the intervention group as compared to the control group. Three possible mechanisms have demonstrated that vitamin $\mathrm{D}_{3}$ may affect muscle function $(33,34)$. First, it may act by controlling ionized calcium concentrations; thus, calcium can have a direct and primary effect on muscle cell function. Second, it may have a direct effect on dividing myoblasts, and make differentiation to multinucleated non-dividing myotubes. Finally, it may have a direct effect on muscle cells to make expression of specific genes, i.e. a vitamin Ddependent Ca-binding protein or specific $\mathrm{Ca}^{++}$ ATPase enzyme; therefore, changing calcium control of the muscle cells. Furthermore, vitamin D deficiency leads to myopathies and to disruptions of balance and neuromuscular coordination (35). An improvement in neuromuscular coordination impairment leads to nerve growth factor synthesis by vitamin $\mathrm{D}$ derivatives as shown in vitro in fibroblasts and astrocytes and in vivo in rat brain (36). This shows that vitamin $\mathrm{D}$ deficiency influences more coordinative muscle function than muscle strength.

At the end of the intervention, fat mass decreased in the vitamin D group and increased in the placebo group, while no significant differences were seen between the two groups. In some cross-sectional studies (36-38), 25(OH)-D concentrations correlated negatively with sarcopenia. However, cross-sectional design could not demonstrate the causality of vitamin $\mathrm{D}$ and sarcopenia. It is possible that sarcopenia leads to low vitamin D status. Firstly, sarcopenia has proven to be a strong predictor of disablement and mortality in older persons (38). Declined physical activity and less time spent outdoors lead to vitamin D insufficiency. Secondly, sarcopenia is mainly observed in obese persons, who demonstrate significant muscle loss despite an elevated fat mass (37). Elevated body fat mass causes trapping of vitamin $\mathrm{D}$ in the adipose tissue, and lowers the serum concentration of vitamin D (38). Third, sarcopenia is associated with aging (37). Old age is a risk factor for vitamin $\mathrm{D}$ insufficiency because the cutaneous synthesis of vitamin $\mathrm{D}_{3}$ decreases with age (31). In addition, serum $25(\mathrm{OH})$-D concentration is an important determinant of increased PTH level that promotes calcium influx in to the adipocytes (37), where intracellular calcium promotes lipogenesis. PTH excess may lead to weight gain, and may be associated with adiposity (37).

There were some limitations in the present study; firstly, the duration of vitamin D supplementation was short, and secondly, serum PTH levels were not measured. The long term association between vitamin $\mathrm{D}$ intake and muscle strength regarding its relationship with PTH is also needed to be investigated.

It seems that $1000 \mathrm{IU}$ vitamin D consumption, in the vitamin D-deficient middle-aged women group daily for 12 weeks, resulted in improvement in muscle function and body composition; however, it had no significant effect on muscle strength.

\section{Acknowledgement}

We would like to convey our appreciation to the staff of Iran University of Medical Sciences (IUMS) for their cooperation and participation in this study.

\section{Financial disclosure}

None of the authors have any conflict of interest to declare.

\section{Funding/Support}

This work was financially supported by the Vice Chancellor of Research, Iran University of Medical Sciences (Tehran, Iran).

\section{References}

1. Lauretani F, Russo C, Bandinelli S, Bartali B, Cavazzini $\mathrm{C}$, Iorio A, et al. Age associated changes in skeletal muscles and their effect on mobility: An operational diagnosis of sarcopenia. J Appl Physiol 2003; 95:185160 . 
2. Greenlund LJS, Nair KS. Sarcopenia-consequences, mechanisms, and potential therapies. Mechanisms of Ageing and Development 2003; 124:287-99.

3. Doherty T -Invited Review-: Aging and sarcopenia. J Appl Physiol 2003; 95:1717-27.

4. Robinson S, Cooper C, Aihie Sayer A. Nutrition and sarcopenia: A review of the evidence and implications for preventive strategies. J Aging Res 2012; 510801.

5. Robinson SM, Jameson KA, Batelaan SF, Martin HJ, Syddall HE, Dennison EM, et al. Diet and its relationship with grip strength in community-dwelling older men and women: The Hertfordshire cohort study. J Am Geriatr Soc 2008; 56:84-90.

6. Park, S., J.-O. Ham, and B.-K. Lee, A positive association of vitamin D deficiency and sarcopenia in 50 year old women, but not men. Clinical Nutrition, 2013.

7. Haroon M, FitzGerald O. Vitamin D deficiency: Subclinical and clinical consequences on musculoskeletal health. Curr Rheumatol Rep 2012; 14:286-93.

8. Scott D, Blizzard L, Fell J, Ding C, Winzenberg T, Jones G. A prospective study of the associations between 25hydroxy-vitamin $\mathrm{D}$, sarcopenia progression and physical activity in older adults. Clin Endocrinol (Oxf) 2010; 73:581-87.

9. Schott GD, Wills MR. Muscle weakness in osteomalacia. Lancet 1976; 1:626-9.

10. Gloth FM III, Gundberg CM, Hollis BW, Haddad JG,Tobin JD. Vitamin D deficiency in homebound elderly persons. JAMA 1995; 274: 1683-86.

11. Omdahl JL, Garry PJ, Hunsaker LA, Hunt WC, Goodwin JS. Nutritional status in a healthy elderly population: Vitamin D. Am J Clin Nutr 1982; 36:122533.

12. McKenna MJ. Differences in vitamin D status between countries in young adults and the elderly. Am J Med 1992; 93:69-77.

13. Holick MF. Environmental factors that influence the cutaneous production of vitamin D. Am J Clin Nutr 1995; 61(suppl):638S-45S.

14. Janssen H, M M samson, and H J Verhaar, Vitamin D deficiency, muscle function, and falls in elderly people. Am J Clin Nutr 2002; 75:611-15.

15. Evans W. Functional and metabolic consequences of sarcopenia. The Journal of nutrition 1997; 127(5 Suppl):998S-1003S.

16. Nemere I, Schwartz Z, Pedrozo H, Sylvia VL, Dean $\mathrm{DD}$, Boyan BD. Identification of a membrane receptor for 1,25-dihydroxyvitamin D3 which mediates rapid activation of protein kinase C. J Bone Miner Res 1998; 13:1353-59.

17. Massheimer V, Fernandez LM, Boland R, de Boland AR. Regulation of $\mathrm{Ca}^{2+}$ uptake in skeletal muscle by 1,25-dihydroxyvitamin D3: Role of phosphorylation and calmodulin. Mol Cell Endocrinol 1992; 84: 15-22.

18. De Boland AR, Boland RL. Non-genomic signal transduction pathway of vitamin $\mathrm{D}$ in muscle. Cell Signal 1994; 6:717-24.

19. Vazquez G, de Boland AR, Boland R. Stimulation of $\mathrm{Ca}^{2+}$ release- activated $\mathrm{Ca}^{2+}$ channels as a potential mechanism involved in non- genomic 1,25(OH)2vitamin D3-induced $\mathrm{Ca}^{2+}$ entry in skeletal muscle cells. Biochem Biophys Res Commun 1997; 239:562-65.

20. Snijder MB, R.M Dam, M Visser, D.J.H Deeg, J.M Dekker, L.M Bouter, et al. Adiposity in Regulation to Vitamin D status and Parathyroid Hormone Levels: A Population-Based Study in Older Men and Women. J Clin Endocrinol Metab 2005; 90: 4119-23.

21. Luna-Heredia E, Martin-Pena G, Ruiz-Galiana J. Handgrip dynamometry in healthy adults. Clin Nutr $2005 ; 24: 250-258$.

22. Craig CL, Marshall AL, Sjostrom M, Bauman AE, Booth ML, Ainsworth BE, et al. International physical activity questionnaire: 12-country reliability and validity. Med Sci Sports Exerc 2003; 35:1381-95.

23. Bahareh N, Tirang R N, Maryam S F, Hamid AM, Anahita H, Ali K, et al. Daily consumption of vitamin D- or vitamin D + calcium-fortified yogurt drink improved glycemic control in patients with type 2 diabetes: A randomized clinical trial. Am J Clin Nutr 2011; 93:764-71.

24. Budziareck MB, Pureza Duarte RR, Barbosa-Silva MC. Reference values and determinants for handgrip strength in healthy subjects. Clin Nutr 2008; 27:357-62.

25. Rolland Y, Lauwers-Cances V, Pahor M, Fillaux J, Grandjean H, Vellas B. Muscle strength in obese elderly women: Effect of recreational physical activity in a cross-sectional study. Am J Clin Nutr 2004; 79:552-57.

26. Samson MM, Meeuwsen IB, Crowe A, Dessens JA, Duursma SA, Verhaar HJ. Relationships between physical performance measures, age, height and body weight in healthy adults. Age Ageing 2000; 29:235-42.

27. Wall JC, Bell C, Campbell S, Davis J. The Timed Getupand- Go test revisited: measurement of the component tasks. J Rehabil Res Dev 2000; 37:109-13.

28. Hennie C.J.P. Janssen, Monique M. Samson and Harald J.J. Verhaar. Muscle strength and mobility in vitamin D-insufficient female geriatric patients: A randomized controlled trial on vitamin D and calcium supplementation. Aging Clin Exp Res 2010; 22: 78-84.

29. Holick MF. Vitamin D deficiency. N Engl J Med 2007; 357:266-81.

30. Bischoff-Ferrari HA, Dawson-Hughes B, Staehelin HB et al. Fall prevention with supplemental and active forms of vitamin D: A meta-analysis of randomised controlled trials. BMJ 2009; 339:b3692. 
31. L. Ceglia. Vitamin D and skeletal muscle tissue and function. Molecular Aspects of Medicine 2008; 29: 407-14.

32.Redzic M, Regina M. Lewis, D. Travis Thomas. Relationship between 25 hydoxyvitamin D, muscle strength, and incidence of injury in healthy adults: A systematic review. Nutrition Research 2013; 33: 25158.

33. Simpson R, Thomas G, Arnold A. 1,25 Dihydroxyvitamin D receptors in skeletal and heart muscle cells. J. Biol. Chem 1985; 260: 8882-84.

34. Boland R. Role of vitamin D in skeletal muscle function. Endocr. Rev 1986; 7: 434-43.

35. Micheal P, Bettina B, Helmut W. M, Christine A, Detlef N, and Corinna H. Effects of a Short-Term Vitamin D and Calcium Supplementation on Body
Sway and Secondary Hyperparathyroidism in Elderly Women. J Bone Miner Res 2000; 15:1113-18.

36. Sunmin Park, Jung-O Ham, Byung-Kook Lee. A positive association of vitamin $\mathrm{D}$ deficiency and sarcopenia in 50 year old women, but not men. Clinical Nutrition 2014; 33:900-905.

37. Mee Kyoung Kim, Ki Hyun Baek, Ki-Ho Song, Moo Il Kang, Cheol Young Park, Won Young Lee, et al. Vitamin D Deficiency Is Associated with Sarcopenia in Older Koreans, Regardless of Obesity: The Fourth Korea National Health and Nutrition Examination Surveys (KNHANES IV) 2009. J Clin Endocrinol Metab 2011; 96(10):3250-56.

38. Sonia A, Simcha P, James Y, and John F. A. Body Fat Content and 25-Hydroxyvitamin D Levels in Healthy Women. J Clin Endocrinol Metab 2003; 88: 157-61. 